\title{
ON A TWO-SIDEDLY DEGENERATE CHEMOTAXIS MODEL WITH VOLUME-FILLING EFFECT
}

\author{
MOSTAFA BENDAHMANE, KENNETH H. KARLSEN AND JOSÉ MIGUEL URBANO
}

\begin{abstract}
We consider a fully parabolic model for chemotaxis with volume-filling effect and a nonlinear diffusion that degenerates in a two-sided fashion. We address the questions of existence of weak solutions and of their regularity by using, respectively, a regularization method and the technique of intrinsic scaling.
\end{abstract}

Keywords: Chemotaxis, reaction-diffusion, degenerate PDE, intrinsic scaling. AMS Subject Classification (2000): 35K65, 92C17, 35B65.

\section{Introduction}

Chemotaxis is a property of certain living organisms to be repelled or attracted to chemical substances. In [16, 17], Keller and Segel introduced a model for aggregation of the cellular slime mold Dictyostelium discoideum due to cyclic Adenosine Monophosphate (cAMP), which is an attractive chemical signal for the amoebae.

In this paper, we study the following model for chemotactic movement:

$$
\left\{\begin{array}{l}
\partial_{t} u-\operatorname{div}(a(u) \nabla u-\chi u f(u) \nabla v)=0 \quad \text { in } Q_{T}, \\
\partial_{t} v-d \Delta v=g(u, v) \quad \text { in } Q_{T},
\end{array}\right.
$$

where $Q_{T}:=\Omega \times(0, T), T>0$ is a fixed time, and $\Omega$ is a bounded domain in $\mathbb{R}^{N}$, with smooth boundary $\partial \Omega$ and outer unit normal $\eta$.

We augment (1) with no-flux boundary conditions on $\Sigma_{T}:=\partial \Omega \times(0, T)$,

$$
a(u) \frac{\partial u}{\partial \eta}=0, \quad \frac{\partial v}{\partial \eta}=0,
$$

and initial distributions in $\Omega$ :

$$
u(x, 0)=u_{0}(x), \quad v(x, 0)=v_{0}(x) .
$$

Received February 20, 2006.

This research was supported by an Outstanding Young Investigators Award from the Research Council of Norway. The research of J. M. Urbano was supported by CMUC/FCT and Project POCI/MAT/57546/2004. 
In the chemotaxis model above, $u=u(x, t)$ represents the density of the cell-population, $v=v(x, t)$ represents the chemoattractant (repellent) concentration, $a(u)$ is a density-dependent diffusion coefficient, and $d$ is a constant. Furthermore, $f(u(x, t))$ is a density dependent probability that a cell in position $x$ at time $t$ finds space in its neighboring location. The cells are attracted by the chemical and $\chi$ denotes their chemotactic sensitivity. The function $g(u, v)$ describes the rates of production and degradation of the chemoattractant; here, we assume it is the linear function

$$
g(u, v)=\alpha u-\beta v, \quad \alpha, \beta \geq 0 .
$$

This assumption has also been used in related literature (see, e.g., [12]).

In this work, we assume at first that there exists a maximal density of cells, the threshold $u_{m}$, such that $f\left(u_{m}\right)=0$. Intuitively, this amounts to a switch to repulsion at high densities, sometimes referred to as volume-filling effect or prevention of overcrowding (see [11]). Secondly, we assume that the densitydependent diffusion coefficient $a(u)$ degenerates for $u=0$ and $u=u_{m}$. This means that there is no diffusion when $u$ approaches values close to the threshold (see [20]) and in the absence of cell-population. This interpretation was proposed in [4] where the diffusion coefficient takes the form $a(u)=$ $\varepsilon u(1-u)$, for $\varepsilon>0$. The main advantages of this nonlinear diffusion model seem to be related to the finite speed of propagation (which is more realistic in biological applications than infinite speed) and the asymptotic behavior of solutions (see [4], for a discussion of this and related aspects of the model). The threshold condition has a clear biological interpretation: the cells stop to accumulate at a given point of $\Omega$ after their density attains certain threshold values and the chemotactic cross diffusion $h(u)=\chi u f(u)$ vanishes identically when $u \geq u_{m}$.

Defining new variables through

$$
\tilde{u}=\frac{u}{u_{m}}, \quad \tilde{v}=v
$$

we have $\tilde{u}_{m}=1$. After performing this linear transformation, we may thus omit the tildes in the notation and assume from now on, without loss of generality, that $u_{m}=1$. A typical example of $f$ in this case is

$$
f(u)=1-u \text {. }
$$

More generally, in this work we assume that the function $f$ in (1) satisfies

$$
f \in C^{2}([0,1]) \text { and } f(1)=0 .
$$


Regarding the nonlinear diffusion coefficient $a$, we assume that

$$
a \in C^{1}([0,1]), a(0)=a(1)=0 \text { and } a(s)>0 \text { for } 0<s<1 .
$$

This assumption will be used to prove the existence of weak solutions. To ensure that the weak solutions are Hölder continuous, further assumptions on $a$ will be needed, namely that there exists a number $\delta \in(0,1 / 2)$ such that for some constants $1<C_{0} \leq C_{1}$

$$
\begin{aligned}
& C_{0} \phi_{1}(s) \leq a(s) \leq C_{1} \phi_{1}(s), \text { for all } s \in[0, \delta] \\
& C_{0} \phi_{2}(1-s) \leq a(s) \leq C_{1} \phi_{2}(1-s), \text { for all } s \in[1-\delta, 1]
\end{aligned}
$$

where $\phi_{1}(s)=s^{p_{1}}$ and $\phi_{2}(s)=s^{p_{2}}$ for some $0<p_{1}<p_{2}$. A typical example of a function satisfying (6) is provided by any $a \in C^{1}$ for which

$$
a(s)=C s^{p_{1}} \text { for } s \in[0, \delta] \text { and } a(s)=C(1-s)^{p_{2}} \text { for } s \in[1-\delta, 1]
$$

for some constant $C>1$.

The case when $a \equiv 1, f \equiv 1$ in (1) has been the object of extensive research; the existence and uniqueness of solutions, as well as their asymptotic behavior, have been studied by several authors. In [27], the author obtained the local in time existence of a unique, positive, classical solution, together with results on the blow-up behavior of solutions. In [22], the existence of an exponential attractor is proved for the corresponding dynamical system. In [10], the authors proved in the case $\Omega \subset \mathbb{R}^{2}$ is a disk, $u_{0}, v_{0}$ are radial functions, and $\left\|u_{0}\right\|_{L^{1}}$ is sufficiently large, that $\|u(t)\|_{L^{2}}$ blows up in finite time, hence that (1) does not admit a global solution in this case.

Assuming that $a$ is degenerate only at one point $(u=1)$, existence and uniqueness results were established recently by Laurençot and Wrzosek [20] using the abstract theory developed in [2]. To contrast with the work [20], we prove herein the existence of weak solutions for the degenerate system (1)-(3), which has a two-point degeneracy $(u=0,1)$, using a Schauder fixed-point argument on a regularized problem and the compactness method. Additionally, we prove that these weak solutions are in fact Hölder continuous.

Before stating our main results, we give the definition of a weak solution. 
Definition 1. A weak solution of (1)-(3) is a pair $(u, v)$ of functions satisfying the following conditions:

$$
\begin{aligned}
& 0 \leq u(x, t) \leq 1 \text { and } v(x, t) \geq 0, \text { for a.e. }(x, t) \in Q_{T}, \\
& u \in C_{w}\left(0, T, L^{2}(\Omega)\right), \quad \partial_{t} u \in L^{2}\left(0, T ;\left(H^{1}(\Omega)\right)^{\prime}\right), \quad u(0)=u_{0}, \\
& A(u):=\int_{0}^{u} a(s) d s \in L^{2}\left(0, T ; H^{1}(\Omega)\right), \\
& v \in L^{\infty}\left(Q_{T}\right) \cap L^{p}\left(0, T ; W^{2, p}(\Omega)\right) \cap C\left(0, T, L^{2}(\Omega)\right), \text { for all } p>1, \\
& \partial_{t} v \in L^{2}\left(Q_{T}\right), \quad v(0)=v_{0},
\end{aligned}
$$

and, for all $\varphi, \psi \in L^{2}\left(0, T ; H^{1}(\Omega)\right)$,

$$
\begin{aligned}
& \int_{0}^{T}\left\langle\partial_{t} u, \varphi\right\rangle d t+\iint_{Q_{T}} a(u) \nabla u \cdot \nabla \varphi d x d t-\iint_{Q_{T}} \chi u f(u) \nabla v \cdot \nabla \varphi d x d t=0, \\
& \iint_{Q_{T}} \partial_{t} v \psi d x d t+\iint_{Q_{T}} d \nabla v \cdot \nabla \psi d x d t=\iint_{Q_{T}} g(u, v) \psi d x d t
\end{aligned}
$$

where $C_{w}\left(0, T, L^{2}(\Omega)\right)$ denotes the space of continuous functions with values in (a closed ball of) $L^{2}(\Omega)$ endowed with the weak topology, and $\langle\cdot, \cdot\rangle$ denotes the duality pairing between $H^{1}(\Omega)$ and $\left(H^{1}(\Omega)\right)^{\prime}$.

Our first result is the following existence theorem for weak solutions.

Theorem 1. Assume (4) and (5) hold. If $u_{0}, v_{0} \in L^{\infty}(\Omega)$ with $0 \leq u_{0} \leq 1$ and $v_{0} \geq 0$ a.e. in $\Omega$, then there exists a weak solution to the degenerate system (1)-(3) in the sense of Definition 1.

A major difficulty for the analysis of the system (1)-(3) is the strong degeneracy of the diffusion term. To handle this difficulty, we replace the original diffusion term $a(u)$ by $a_{\varepsilon}(u)=a(u)+\varepsilon$ and consider, for each fixed $\varepsilon>0$, the non-degenerate problem

$$
\begin{aligned}
& \partial_{t} u_{\varepsilon}-\operatorname{div}\left(a_{\varepsilon}\left(u_{\varepsilon}\right) \nabla u_{\varepsilon}-\chi u_{\varepsilon} f\left(u_{\varepsilon}\right) \nabla v_{\varepsilon}\right)=0 \quad \text { in } Q_{T}, \\
& \partial_{t} v_{\varepsilon}-d \Delta v_{\varepsilon}=g\left(u_{\varepsilon}, v_{\varepsilon}\right) \quad \text { in } Q_{T}, \\
& a_{\varepsilon}\left(u_{\varepsilon}\right) \frac{\partial u_{\varepsilon}}{\partial \eta}=0, \quad \frac{\partial v_{\varepsilon}}{\partial \eta}=0 \quad \text { on } \Sigma_{T}, \\
& u_{\varepsilon}(x, 0)=u_{0}(x), \quad v_{\varepsilon}(x, 0)=v_{0}(x) \quad \text { for } x \in \Omega .
\end{aligned}
$$


To prove Theorem 1 we first prove existence of solutions to the non-degenerate problem (7) by applying the Schauder fixed-point theorem (in an appropriate functional setting). Then we send the regularization parameter $\varepsilon$ to zero to produce a weak solution of the original system (1)-(3) as the limit of a sequence of such approximate solutions. Convergence is achieved by means of a priori estimates and compactness arguments.

Our second result is the following regularity theorem for weak solutions.

Theorem 2. Under assumptions (4)-(6), any weak solution $u$ of the system (1)-(3) is locally Hölder continuous.

The proof of Theorem 2 is based upon the use of intrinsic scaling, a powerful technique in the framework of regularity theory for degenerate and singular PDEs. Continuity results for weak solutions follow from a fine analysis of the behaviour of their oscillation in a sequence of nested and shrinking cylinders, namely showing that it converges to zero. When it is possible to determine quantitatively how this happens a modulus of continuity is derived. Intrinsic scaling amounts to analyze each equation in a geometrical framework related to its structure, in the sense that the iteration process just described must be developed in cylinders whose dimensions have to be rescaled according to the degeneracies of the PDE. This will reflect the fact that the diffusion processes in the equation evolve in a time scale determined instant by instant by the solution itself, so that, loosely speaking, it can be regarded as the heat equation in its own intrinsic time-configuration. For a modern account of the theory and related matters, see the updated survey [7]; recent applications in the context of the modelling of phase transitions or the porous media equation with variable exponent of nonlinearity are to be found in [8] and [9], respectively.

The same type of two-sided degeneracy treated here occurs in a model for the flow of two immiscible fluids in a porous medium (cf. [1] and the references therein). The results obtained build upon the analysis developed in [26] for this model, where it is shown that weak solutions are locally Hölder continuous if $a(\cdot)$ decays like a power at both degeneracies. The novelty in this work is the additional lower-order term $\operatorname{div}(\chi u f(u) \nabla v)$. It is shown that this term satisfies the appropriate growth conditions due to its special form and the available regularity for $v$.

We have not been able to prove uniqueness for our model because of the presence of a nonlinear degenerate diffusion term and a nonlinear transport 
term. For their one-point degenerate model, Laurençot and Wrzosek overcome this kind of difficulty in [20] by imposing the following condition:

$$
\left(f(u)+u f^{\prime}(u)\right)^{2} \leq C_{2} a(u), \forall u>0, \quad \text { for some constant } C_{2}>0 .
$$

In this case, uniqueness can be proved using a duality approach. As already mentioned, in [20] the diffusion term $a$ vanishes only at the threshold value $u=1$, and typical examples of $a$ and $f$ are

$$
a(s)=(1-s)^{n} \text { and } f(s)=s(1-s)^{m},
$$

for $m \geq 1+n / 2$ and $m \geq 2$.

A successful technique for proving uniqueness of (entropy weak) solutions to degenerate parabolic equations is based on Kružkov's method [23] for obtaining the $L^{1}$ contraction property of scalar conservation laws, see Carrillo [5] and also [14, 3]. Related to this approach we want to mention that in [4] the authors prove, among other things, a uniqueness result for a degenerate parabolic-elliptic system set in an unbounded domain. The method used in [4], which relies on a continuous dependence estimate from [14], does not apply to our problem, because it is difficult to bound $\Delta v$ in $L^{\infty}\left(Q_{T}\right)$ due to the parabolic nature of the $v$-equation in (1)-(3).

The plan of the paper is as follows: in Section 2 we prove existence of solutions to the non-degenerate problem; the main results are proved in Section 3 (Theorem 1) and Section 4 (Theorem 2).

\section{Existence of solutions for the non-degenerate problem}

In this section we prove, for each fixed $\varepsilon>0$, the existence of solutions to the non-degenerate problem (7), by applying the Schauder fixed-point theorem. For this purpose, we introduce the following closed subset of the Banach space $L^{2}\left(Q_{T}\right)$ :

$$
\mathcal{K}=\left\{u \in L^{2}\left(Q_{T}\right): 0 \leq u(x, t) \leq 1 \text {, for a.e. }(x, t) \in Q_{T}\right\} .
$$

In this section, we omit the dependence of the solutions on the parameter $\varepsilon$.

2.1. Weak solution to the non-degenerate problem. With $\bar{u} \in \mathcal{K}$ fixed, let $v$ be the unique solution of the parabolic problem

$$
\left\{\begin{array}{l}
\partial_{t} v-d \Delta v=g(\bar{u}, v), \quad \text { in } Q_{T} \\
\frac{\partial v}{\partial \eta}=0 \text { on } \Sigma_{T}, \quad v(x, 0)=v_{0}(x) \text { for } x \in \Omega .
\end{array}\right.
$$


Given the function $v$, let $u$ be the unique solution of the quasilinear parabolic problem

$$
\left\{\begin{array}{l}
\partial_{t} u-\operatorname{div}\left(a_{\varepsilon}(u) \nabla u-\chi u f(u) \nabla v\right)=0, \quad \text { in } Q_{T} \\
a_{\varepsilon}(u) \frac{\partial u}{\partial \eta}=0 \text { on } \Sigma_{T}, \quad u(x, 0)=u_{0}(x) \text { for } x \in \Omega .
\end{array}\right.
$$

In (9) - (10), $v_{0}$ and $u_{0}$ are functions satisfying the hypothesis of Theorem 1.

Note that for any fixed $\bar{u} \in \mathcal{K}$, problem (9) is uniformly parabolic, so we have immediately the following lemma (see [18]).

Lemma 1. If $v_{0} \in L^{\infty}(\Omega)$, then (9) has a unique solution $v \in L^{\infty}\left(Q_{T}\right) \cap$ $L^{p}\left(0, T ; W^{2, p}(\Omega)\right) \cap C\left(0, T ; L^{2}(\Omega)\right)$, for all $p>1$, satisfying in particular

$$
\begin{aligned}
& \|v\|_{L^{\infty}\left(Q_{T}\right)}+\|v\|_{L^{\infty}\left(0, T ; L^{2}(\Omega)\right)} \leq C, \\
& \|v\|_{L^{2}\left(0, T ; H^{1}(\Omega)\right)} \leq C, \\
& \left\|\partial_{t} v\right\|_{L^{2}\left(Q_{T}\right)} \leq C,
\end{aligned}
$$

where $C>0$ is a constant which depends only on $\left\|v_{0}\right\|_{L^{\infty}(\Omega)}, \alpha, \beta$, and $\operatorname{meas}\left(Q_{T}\right)$.

Regarding the quasilinear problem (10) we have the following lemma (see [18]):

Lemma 2. If $u_{0} \in L^{\infty}(\Omega)$, then, for any $\varepsilon>0$, there exists a unique weak solution $u \in L^{\infty}\left(Q_{T}\right) \cap L^{2}\left(0, T ; H^{1}(\Omega)\right)$ to problem $(10)$.

We establish in the next subsection a fixed-point theorem for (9)-(10) by introducing a map $\Theta: \mathcal{K} \rightarrow \mathcal{K}$ such that $\Theta(\bar{u})=u$, where $u$ solves (10), i.e., $\Theta$ is the solution operator of (10) associated with the coefficient $\bar{u}$ and the solution $v$ coming from (9).

2.2. The fixed-point method. By using the Schauder fixed-point theorem, we prove that the map $\Theta$ has a fixed point. First, let us show that $\Theta$ is a continuous mapping. Let $\left(\bar{u}_{n}\right)_{n}$ be a sequence in $\mathcal{K}$ and $\bar{u} \in \mathcal{K}$ be such that $\bar{u}_{n} \rightarrow \bar{u}$ in $L^{2}\left(Q_{T}\right)$ as $n \rightarrow \infty$. Define $u_{n}=\Theta\left(\bar{u}_{n}\right)$, i.e., $u_{n}$ is the solution of (10) associated with $\bar{u}_{n}$ and the solution $v_{n}$ of (9). The objective is to show that $u_{n}$ converges to $\Theta(\bar{u})$ in $L^{2}\left(Q_{T}\right)$. We start with the following lemma:

Lemma 3. The solutions $u_{n}$ to problem (10) satisfy

(i) $0 \leq u_{n}(x, t) \leq 1$, for a.e. $(x, t) \in Q_{T}$. 
(ii) The sequence $\left(u_{n}\right)_{n}$ is bounded in $L^{2}\left(0, T ; H^{1}(\Omega)\right) \cap L^{\infty}\left(0, T ; L^{2}(\Omega)\right)$.

(iii) The sequence $\left(u_{n}\right)_{n}$ is relatively compact in $L^{2}\left(Q_{T}\right)$.

Proof: (i) We introduce the function $h(s)=s f(s)$, for $s \in \mathbb{R}$, and choose a Lipschitz continuous extension $\tilde{h}$ of $h$ satisfying

$$
\begin{gathered}
\tilde{h}(s)=s f(s) \text { if } 0 \leq s \leq 1, \tilde{h}(s)=h(0)=0 \text { if } s \leq 0, \\
\text { and } \tilde{h}(s)=h(1)=0 \text { if } s \geq 1 .
\end{gathered}
$$

We then replace the equation in (10) by

$$
\partial_{t} u_{n}-\operatorname{div}\left(a_{\varepsilon}\left(u_{n}\right) \nabla u_{n}-\chi \tilde{h}\left(u_{n}\right) \nabla v_{n}\right)=0, \quad \text { in } Q_{T} .
$$

Multiplying (12) by $-u_{n}^{-}=\frac{u_{n}-\left|u_{n}\right|}{2}$ and integrating over $\Omega$, we get

$$
\frac{1}{2} \frac{d}{d t} \int_{\Omega}\left|u_{n}^{-}\right|^{2} d x+\int_{\Omega} a_{\varepsilon}\left(u_{n}\right) \nabla u_{n}^{-} \cdot \nabla u_{n}^{-} d x=\chi \int_{\Omega} \tilde{h}\left(u_{n}\right) \nabla v_{n} \cdot \nabla u_{n}^{-} d x .
$$

Since $\tilde{h}(s)=0$ for $s \leq 0$ and according to the positivity of the second term of the left-hand side, we obtain

$$
\frac{1}{2} \frac{d}{d t} \int_{\Omega}\left|u_{n}^{-}\right|^{2} d x \leq 0
$$

Next, we multiply $(12)$ by $\left(u_{n}-1\right)^{+}$and integrate over $\Omega$. The result is

$$
\begin{array}{r}
\frac{1}{2} \frac{d}{d t} \int_{\Omega}\left|\left(u_{n}-1\right)^{+}\right|^{2} d x+\int_{\Omega} a_{\varepsilon}\left(u_{n}\right) \nabla\left(u_{n}-1\right)^{+} \cdot \nabla\left(u_{n}-1\right)^{+} d x \\
=\chi \int_{\Omega} \tilde{h}\left(u_{n}\right) \nabla v_{n} \cdot \nabla\left(u_{n}-1\right)^{+} d x .
\end{array}
$$

Since $\tilde{h}(s)=0$ for $s \geq 1$, we obtain from (13)

$$
\frac{d}{d t} \int_{\Omega}\left|\left(u_{n}-1\right)^{+}\right|^{2} d x \leq 0 .
$$

Using that $u_{0} \leq 1$ in $\Omega$, we conclude from this $u_{n}(t, \cdot) \leq 1$ in $\Omega$ for all $t \in(0, T)$.

(ii) We multiply the equation (12) by $u_{n}$ and integrate over $\Omega$ to obtain

$$
\frac{1}{2} \frac{d}{d t} \int_{\Omega}\left|u_{n}\right|^{2} d x+\int_{\Omega} a_{\varepsilon}\left(u_{n}\right)\left|\nabla u_{n}\right|^{2} d x=\chi \int_{\Omega} u_{n} f\left(u_{n}\right) \nabla v_{n} \cdot \nabla u_{n} d x .
$$


An integration by parts, exploiting the zero Neumann boundary condition for $v$ and the boundedness of $u$, reveals that the right-hand side is bounded independently of $n$, so that

$$
\frac{1}{2} \frac{d}{d t} \int_{\Omega}\left|u_{n}\right|^{2} d x+\varepsilon \int_{\Omega}\left|\nabla u_{n}\right|^{2} d x \leq C,
$$

for some constant $C$ independent of $n$. This completes the proof of (ii).

Finally, (iii) is a consequence of (ii) and the uniform boundedness of $\left(\partial_{t} u_{n}\right)_{n}$ in $L^{2}\left(0, T ;\left(H^{1}(\Omega)\right)^{\prime}\right)$.

The following lemma contains a classical result (see [18]).

Lemma 4. There exists a function $v \in L^{2}\left(0, T ; H^{1}(\Omega)\right)$ such that the sequence $\left(v_{n}\right)_{n}$ converges strongly to $v$ in $L^{2}\left(0, T ; H^{1}(\Omega)\right)$.

Summarizing our findings so far, from Lemmata 2, 3 and 4, there exist functions $u, v \in L^{2}\left(0, T ; H^{1}(\Omega)\right)$ such that, up to extracting subsequences if necessary,

$$
u_{n} \rightarrow u \text { in } L^{2}\left(Q_{T}\right) \text { strongly, } \quad v_{n} \rightarrow v \text { in } L^{2}\left(0, T ; H^{1}(\Omega)\right) \text { strongly, }
$$

and from this the continuity of $\Theta$ on $\mathcal{K}$ follows.

We observe that, from Lemma $3, \Theta(\mathcal{K})$ is bounded in the set

$$
\mathcal{W}=\left\{u \in L^{2}\left(0, T ; H^{1}(\Omega)\right): u \in L^{2}\left(0, T ;\left(H^{1}(\Omega)\right)^{\prime}\right)\right\} .
$$

By the results of $[25], \mathcal{W} \hookrightarrow L^{2}\left(Q_{T}\right)$ is compact, thus $\Theta$ is compact. Now, by the Schauder fixed point theorem, the operator $\Theta$ has a fixed point $u_{\varepsilon}$ such that $\Theta\left(u_{\varepsilon}\right)=u_{\varepsilon}$. This implies that there exists a solution $\left(u_{\varepsilon}, v_{\varepsilon}\right)$ of

$$
\begin{gathered}
\int_{0}^{T}\left\langle\partial_{t} u_{\varepsilon}, \varphi\right\rangle d t+\iint_{Q_{T}} a_{\varepsilon}\left(u_{\varepsilon}\right) \nabla u_{\varepsilon} \cdot \nabla \varphi d x d t \\
-\iint_{Q_{T}} \chi u_{\varepsilon} f\left(u_{\varepsilon}\right) \nabla v_{\varepsilon} \cdot \nabla \varphi d x d t=0 \\
\iint_{Q_{T}} \partial_{t} v_{\varepsilon} \psi d x d t+\iint_{Q_{T}} d \nabla v_{\varepsilon} \cdot \nabla \psi d x d t=\iint_{Q_{T}} g\left(u_{\varepsilon}, v_{\varepsilon}\right) \psi d x d t
\end{gathered}
$$

for all $\varphi, \psi \in L^{2}\left(0, T ; H^{1}(\Omega)\right)$. 


\section{Existence of weak solutions}

We have shown in Section 2 that the non-degenerate problem (7) admits a solution $\left(u_{\varepsilon}, v_{\varepsilon}\right)$. The goal in this section is to send the regularization parameter $\varepsilon$ to zero in sequences of such solutions to obtain weak solutions of the original system (1)-(3). Note that, for each fixed $\varepsilon>0$, we have shown the existence of a solution $\left(u_{\varepsilon}, v_{\varepsilon}\right)$ to $(7)$ such that

$$
0 \leq u_{\varepsilon}(x, t) \leq 1 \quad \text { and } \quad 0 \leq v_{\varepsilon}(x, t),
$$

for a.e. $(x, t) \in Q_{T}$.

First, using the second equation of (7) and (18), it is easy to see that the first two estimates of (11) are independent of $\varepsilon$ :

$$
\begin{aligned}
& \left\|v_{\varepsilon}\right\|_{L^{\infty}\left(Q_{T}\right)}+\left\|v_{\varepsilon}\right\|_{L^{\infty}\left(0, T ; L^{2}(\Omega)\right)} \leq C, \\
& \left\|v_{\varepsilon}\right\|_{L^{2}\left(0, T ; H^{1}(\Omega)\right)} \leq C,
\end{aligned}
$$

where $C>0$ is a constant not depending on $\varepsilon$.

Note that, from (18) and (19), the right-hand side of the second equation in the system (7) is bounded. Therefore, from classical results on $L^{p}$ regularity, we have

$$
\left\|\partial_{t} v_{\varepsilon}\right\|_{L^{p}\left(Q_{T}\right)}+\left\|v_{\varepsilon}\right\|_{L^{p}\left(0, T ; W^{2, p}(\Omega)\right)} \leq C, \quad 1 \leq p<\infty
$$

where $C>0$ is a constant independent of $\varepsilon$.

Let $A_{\varepsilon}(s)=A(s)+\varepsilon s$ and $\mathcal{A}(s)=\int_{0}^{s} A(r) d r$. Taking $\varphi=A_{\varepsilon}\left(u_{\varepsilon}\right)$ as a test function in (16), we obtain

$$
\begin{aligned}
& \sup _{0 \leq t \leq T} \int_{\Omega} \mathcal{A}\left(u_{\varepsilon}\right)(x, t) d x+\iint_{Q_{T}}\left|\nabla A\left(u_{\varepsilon}\right)\right|^{2} d x d t \\
& \quad+\varepsilon \sup _{0 \leq t \leq T} \int_{\Omega} \frac{\left|u_{\varepsilon}(x, t)\right|^{2}}{2} d x+\varepsilon \iint_{Q_{T}}\left|\nabla u_{\varepsilon}\right|^{2} d x d t \leq C,
\end{aligned}
$$

for some constant $C>0$ independent of $\varepsilon$, where we have used (19) and the uniform $L^{\infty}$ bound on $u_{\varepsilon}$.

Let $\varphi \in L^{2}\left(0, T ; H^{1}(\Omega)\right)$. Using the weak formulation (16), (19), and (20), we obtain

$$
\begin{aligned}
& \left|\int_{0}^{T}\left\langle\partial_{t} u_{\varepsilon}, \varphi\right\rangle d t\right| \\
& \leq\left\|\nabla A\left(u_{\varepsilon}\right)\right\|_{L^{2}\left(Q_{T}\right)}\|\nabla \varphi\|_{L^{2}\left(Q_{T}\right)}+\left\|\sqrt{\varepsilon} \nabla u_{\varepsilon}\right\|_{L^{2}\left(Q_{T}\right)}\|\nabla \varphi\|_{L^{2}\left(Q_{T}\right)} \\
& \quad+\chi\left\|u_{\varepsilon} f\left(u_{\varepsilon}\right)\right\|_{L^{\infty}\left(Q_{T}\right)}\left\|\nabla v_{\varepsilon}\right\|_{L^{2}\left(Q_{T}\right)}\|\nabla \varphi\|_{L^{2}\left(Q_{T}\right)} \leq C\|\varphi\|_{L^{2}\left(0, T ; H^{1}(\Omega)\right)},
\end{aligned}
$$


for some constant $C>0$ independent of $\varepsilon$. From this we deduce the bound

$$
\left\|\partial_{t} u_{\varepsilon}\right\|_{L^{2}\left(0, T ;\left(H^{1}(\Omega)\right)^{\prime}\right)} \leq C \text {. }
$$

Therefore, by (19) and (20)-(22) and standard compactness results (see [25]) we can extract subsequences, which we do not relabel, such that, as $\varepsilon$ goes to 0 ,

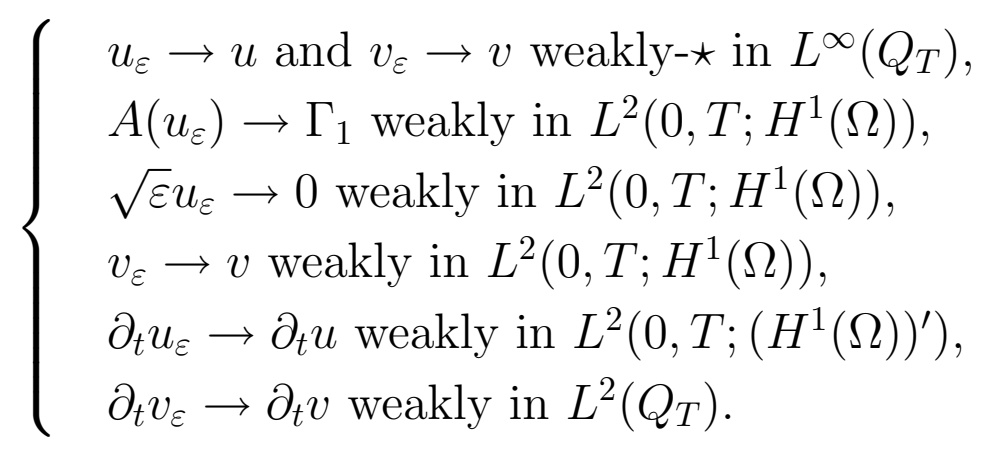

From the compact embedding $L^{\infty}(\Omega) \subset\left(H^{1}(\Omega)\right)^{\prime}$ and according to Corollary 4 of [25]), we also have that $u_{\varepsilon}$ is a Cauchy sequence in $C\left(0, T ;\left(H^{1}(\Omega)\right)^{\prime}\right)$.

It is easy to see that $A\left(u_{\varepsilon}\right)$ is uniformly bounded in $\mathcal{W}$, where $\mathcal{W}$ is defined in (15). Then, from the compact imbedding $\mathcal{W} \subset L^{2}\left(Q_{T}\right)$ we deduce that there exists a subsequence of $u_{\varepsilon}$ such that

$$
A\left(u_{\varepsilon}\right) \rightarrow \Gamma_{1} \text { strongly in } L^{2}\left(Q_{T}\right) .
$$

But as $A$ is monotone, we have $A(u)=\Gamma_{1}$ (see [21]). Thus,

$$
A\left(u_{\varepsilon}\right) \rightarrow A(u) \text { strongly in } L^{2}\left(Q_{T}\right) \text { and a.e. in } Q_{T} .
$$

Furthermore, as $A^{-1}$ is well-defined and continuous, we apply the dominated convergence theorem to $u_{\varepsilon}=A^{-1}\left(A\left(u_{\varepsilon}\right)\right)$ to obtain

$$
u_{\varepsilon} \rightarrow u \text { strongly in } L^{2}\left(Q_{T}\right) \text { and a.e. in } Q_{T} \text {. }
$$

With this and the weak- $\star$ convergence of $u_{\varepsilon}$ to $u$ in $L^{\infty}\left(Q_{T}\right)$, we obtain

$$
u_{\varepsilon} \rightarrow u \text { strongly in } L^{p}\left(Q_{T}\right) \text { for } 1 \leq p<\infty \text {. }
$$

Similarly, $v_{\varepsilon} \rightarrow v$ strongly in $L^{p}\left(Q_{T}\right)$ for $1 \leq p<\infty$.

To pass to the limit in (16) as $\varepsilon \rightarrow 0$, we need the following lemma.

Lemma 5. The sequence $\left(v_{\varepsilon}\right)_{\varepsilon}$ converges strongly to $v$ in $L^{2}\left(0, T ; H^{1}(\Omega)\right)$.

Proof: Subtracting the relations satisfied by $\left(u_{\varepsilon}, v_{\varepsilon}\right)$ and $(u, v)$, we have

$$
\left\{\begin{array}{l}
\partial_{t}\left(v_{\varepsilon}-v\right)-d\left(\Delta v_{\varepsilon}-\Delta v\right)=g\left(u_{\varepsilon}, v_{\varepsilon}\right)-g(u, v) \text { in } Q_{T} \\
\frac{\partial v_{\varepsilon}}{\partial \eta}=\frac{\partial v}{\partial \eta}=0 \text { on } \Sigma_{T}, \quad\left(v_{\varepsilon}-v\right)(x, 0)=0, \text { for } x \in \Omega
\end{array}\right.
$$


Taking $v_{\varepsilon}-v$ as test function in the equation (24) to deduce

$$
\begin{aligned}
\frac{d}{d t} \int_{\Omega}\left|v_{\varepsilon}-v\right|^{2} d x+ & d \int_{\Omega}\left|\nabla\left(v_{\varepsilon}-v\right)\right|^{2} d x \\
& =\int_{\Omega}\left(g\left(u_{\varepsilon}, v_{\varepsilon}\right)-g(u, v)\right)\left(v_{\varepsilon}-v\right) d x .
\end{aligned}
$$

Now using the definition of $g$ and Young's inequality, we deduce from

$$
\begin{aligned}
\frac{d}{d t} \int_{\Omega}\left|v_{\varepsilon}-v\right|^{2} d x+ & d \int_{\Omega}\left|\nabla\left(v_{\varepsilon}-v\right)\right|^{2} d x \\
& \leq \frac{\alpha}{2} \int_{\Omega}\left|u_{\varepsilon}-u\right|^{2} d x+\left(\frac{\alpha}{2}+\beta\right) \int_{\Omega}\left|v_{\varepsilon}-v\right|^{2} d x .
\end{aligned}
$$

Integrating the inequality $(26)$ over $(0, T)$ we obtain

$$
\begin{aligned}
d \iint_{Q_{T}}\left|\nabla\left(v_{\varepsilon}-v\right)\right|^{2} d x d t \leq & \frac{\alpha}{2} \iint_{Q_{T}}\left|u_{\varepsilon}-u\right|^{2} d x d t \\
& +\left(\frac{\alpha}{2}+\beta\right) \iint_{Q_{T}}\left|v_{\varepsilon}-v\right|^{2} d x d t .
\end{aligned}
$$

Finally using the strong convergence in $L^{2}\left(Q_{T}\right)$ of $u_{\varepsilon}$ and $v_{\varepsilon}$ to $u$ and $v$, respectively, we deduce from (27) the strong convergence of the sequence $\left(\nabla v_{\varepsilon}\right)_{\varepsilon}$ to $\nabla v$ in $L^{2}\left(Q_{T}\right)$. This completes the proof.

With the above convergences we are ready to identify the limit $(u, v)$ as a (weak) solution of (1)-(2). Let $\varphi \in L^{2}\left(0, T ; H^{1}(\Omega)\right)$ be a test function in (16). By (23) it is clear that as $\varepsilon \rightarrow 0$

$$
\int_{0}^{T}\left\langle\partial_{t} u_{\varepsilon}, \varphi\right\rangle d t \rightarrow \int_{0}^{T}\left\langle\partial_{t} u, \varphi\right\rangle d t
$$

and

$$
\iint_{Q_{T}} a_{\varepsilon}\left(u_{\varepsilon}\right) \nabla u_{\varepsilon} \cdot \nabla \varphi d x d t \rightarrow \iint_{Q_{T}} a(u) \nabla u \cdot \nabla \varphi d x d t .
$$

Since $h\left(u_{\varepsilon}\right)=u_{\varepsilon} f\left(u_{\varepsilon}\right)$ is bounded in $L^{\infty}\left(Q_{T}\right)$ ( $h$ is continuous), and, by Lemma $5, v_{\varepsilon} \rightarrow v$ in $L^{2}\left(0, T ; H^{1}(\Omega)\right)$, we also have that, as $\varepsilon \rightarrow 0$,

$$
\iint_{Q_{T}} \chi u_{\varepsilon} f\left(u_{\varepsilon}\right) \nabla v_{\varepsilon} \cdot \nabla \varphi d x d t \rightarrow \iint_{Q_{T}} \chi u f(u) \nabla v \cdot \nabla \varphi d x d t
$$


We have thus identified $u$ as the first component of a solution of (1)-(2). Along the same lines, we would identify $v$ as the second component of a solution.

\section{Hölder continuity of weak solutions}

We will concentrate our attention on the interior regularity of $u$; the regularity of $v$ follows from the classical theory of parabolic PDEs. The results extend to the parabolic boundary through standard adaptations of the method $[6,7]$.

Recalling the definition of the Steklov average of a function $w \in L^{1}\left(Q_{T}\right)$

$$
w_{h}=\left\{\begin{array}{cl}
\frac{1}{h} \int_{t}^{t+h} w(\cdot, \tau) \mathrm{d} \tau & \text { if } t \in(0, T-h] \\
0 & \text { if } t \in(T-h, T)
\end{array}, \quad 0<h<T,\right.
$$

the definition of local weak solution for $u$ can be cast in the following formulation, which is technically more convenient and involves the discrete time derivative:

for every subset $K$ of $\Omega$,

$$
\int_{K \times\{t\}}\left\{\partial_{t}\left(u_{h}\right) \varphi+[a(u) \nabla u]_{h} \cdot \nabla \varphi-\chi[u f(u)]_{h} \nabla v \cdot \nabla \varphi\right\} d x=0,
$$

for all $\varphi \in H_{0}^{1}(K)$ and $0<t<T-h$.

Here, $v$ is treated as a given function in its existence class so all the terms in the above expression have a meaning.

4.1. The intrinsic geometry. We study the regularity at the origin $(0,0)$, that we assume to be contained in $Q_{T}$. The result will hold, by translation, for any point $\left(x_{0}, t_{0}\right)$ in the interior of $Q_{T}$. Given a point $x_{0} \in \mathbb{R}^{N}, B_{\rho}\left(x_{0}\right)$ denotes the $N$-dimensional ball with centre at $x_{0}$ and radius $\rho$ :

$$
B_{\rho}\left(x_{0}\right):=\left\{x \in \mathbb{R}^{N}:\left|x-x_{0}\right|<\rho\right\} ;
$$

given a point $\left(x_{0}, t_{0}\right) \in \mathbb{R}^{N+1}$, the cylinder of radius $\rho$ and height $\tau>0$, with vertex at $\left(x_{0}, t_{0}\right)$, is

$$
\left(x_{0}, t_{0}\right)+Q(\tau, \rho):=B_{\rho}\left(x_{0}\right) \times\left(t_{0}-\tau, t_{0}\right) .
$$


We start with the description of the appropriate geometric setting. Consider a small positive number $\epsilon>0$ and $R>0$ such that $Q\left((2 R)^{2-\epsilon}, 2 R\right) \subset Q_{T}$ and define

$\mu_{-}:=\underset{Q\left((2 R)^{2-\epsilon}, 2 R\right)}{\operatorname{ess} \inf } u ; \quad \mu_{+}:=\operatorname{ess~sup}_{Q\left((2 R)^{2-\epsilon}, 2 R\right)} u ; \quad \omega:=\underset{Q\left((2 R)^{2-\epsilon}, 2 R\right)}{\operatorname{ess} \operatorname{Ooc}} u=\mu_{+}-\mu_{-}$.

Construct the cylinder

$$
Q\left(\theta R^{2}, R\right), \quad \text { with } \quad \theta^{-1}=\phi_{1}\left(\frac{\omega}{2^{m}}\right)
$$

where the number $m$ will be chosen large later in the proof, independently of $\omega$. We can take, if need be, $\mu_{+}=1$ and $\mu_{-}=0$, since the other possibilities are clearly more favorable.

We may assume that $Q\left(\theta R^{2}, R\right) \subset Q\left((2 R)^{2-\epsilon}, 2 R\right)$, which means that

$$
-\theta R^{2} \geq-(2 R)^{2-\epsilon} \quad \Leftrightarrow \quad \theta^{-1} \geq 2^{\epsilon-2} R^{\epsilon}
$$

If this does not hold then we have $\phi_{1}\left(\frac{\omega}{2^{m}}\right)<C R^{\epsilon}$, and then the oscillation would go to zero with $R$ and there would be nothing to prove. We then have the relation

$$
\begin{aligned}
& \text { ess OSC } \\
& Q\left(\theta R^{2}, R\right)
\end{aligned}
$$

which will be the starting point of the iteration process leading to the result.

Note that we had to consider the cylinder $Q\left((2 R)^{2-\epsilon}, 2 R\right)$ and assume (29), so that (30) would hold for the rescaled cylinder $Q\left(\theta R^{2}, R\right)$. This is in general not true for a given cylinder since its dimensions would have to be intrinsically defined in terms of the essential oscillation of the function within it. Observe also that when the oscillation $\omega$ is small, and for $m$ very large, then the cylinder $Q\left(\theta R^{2}, R\right)$ is very long in the $t$ direction. It is this feature that will allow us to accommodate the two degeneracies in the problem. We will also assume, without loss of generality, that $\omega<\delta$, where $\delta$ is the number introduced in (6).

We now consider subcylinders of $Q\left(\theta R^{2}, R\right)$ of the form

$$
Q_{R}^{t^{*}} \equiv\left(0, t^{*}\right)+Q\left(\frac{R^{2}}{\phi_{2}\left(\frac{\omega}{4}\right)}, R\right), \quad \text { with } \quad t^{*}<0
$$


They are contained in $Q\left(\theta R^{2}, R\right)$ if $\theta R^{2} \geq-t^{*}+\frac{R^{2}}{\phi_{2}\left(\frac{\omega}{4}\right)}$, which holds if $\phi_{1}\left(\frac{\omega}{2^{m}}\right) \leq$ $\phi_{2}\left(\frac{\omega}{4}\right)$ and $t^{*}$ is chosen such that

$$
t^{*} \in\left(\frac{R^{2}}{\phi_{2}\left(\frac{\omega}{4}\right)}-\frac{R^{2}}{\phi_{1}\left(\frac{\omega}{2^{m}}\right)}, 0\right) .
$$

The result follows from the analysis of an alternative: either there exists a cylinder of the type $Q_{R}^{t^{*}}$ where $u$ is essentially away from its infimum or such a cylinder can not be found. If the former holds then going down to a smaller cylinder the oscillation decreases by a small factor that we can exhibit and that does not depend on the oscillation. In the latter situation then $u$ is essentially away from its supremum in all cylinders of that same type and we can add up this information to reach the same conclusion as in the previous case.

Throughout this section, the letter $C$ denotes a constant that depends only on the data. The same $C$ will be used to denote different constants.

4.2. The alternative. Assume that, for a constant $\nu_{0} \in(0,1)$, that will be determined depending only on the data, there exists a cylinder of the type $Q_{R}^{t^{*}}$ for which

$$
\left|\left\{(x, t) \in Q_{R}^{t^{*}}: u(x, t)>1-\frac{\omega}{2}\right\}\right| \leq \nu_{0}\left|Q_{R}^{t^{*}}\right| .
$$

We start by showing that if (32) holds then $u$ is away from the degeneracy at 1 in a smaller cylinder of the same type. The next lemma specifies what this means.

Lemma 6. There exists a constant $\nu_{0} \in(0,1)$, depending only on the data, such that if (32) holds then

$$
u(x, t)<1-\frac{\omega}{4} \quad \text { a.e. } \quad(x, t) \in Q_{\frac{R}{2}}^{t^{*}} .
$$

Proof: Let $u_{\omega} \equiv \min \left\{u, 1-\frac{\omega}{4}\right\}$. Take the cylinder for which (32) holds, define

$$
R_{n}=\frac{R}{2}+\frac{R}{2^{n+1}}, \quad n=0,1, \ldots,
$$

and construct the family of nested and shrinking cylinders

$$
Q_{R_{n}}^{t^{*}}=B_{R_{n}} \times\left(t^{*}-\frac{R_{n}^{2}}{\phi_{2}\left(\frac{\omega}{4}\right)}, t^{*}\right)
$$


Consider piecewise smooth cutoff functions $0<\xi_{n} \leq 1$, defined in these cylinders, and satisfying the following set of assumptions

$$
\begin{aligned}
& \xi_{n}=1 \text { in } Q_{R_{n+1}}^{t^{*}} ; \quad \xi_{n}=0 \text { on } \partial_{p} Q_{R_{n}}^{t^{*}} \\
& \left|\Delta \xi_{n}\right| \leq \frac{2^{2(n+1)}}{R^{2}} ; \quad\left|\nabla \xi_{n}\right| \leq \frac{2^{n+1}}{R} ; \quad 0 \leq \partial_{t} \xi_{n} \leq 2^{2(n+1)} \frac{\phi_{2}\left(\frac{\omega}{4}\right)}{R^{2}}
\end{aligned}
$$

where $\partial_{p}$ stands for the parabolic boundary, and let

$$
k_{n}=1-\frac{\omega}{4}-\frac{\omega}{2^{n+2}}, \quad n=0,1, \ldots
$$

Choose as test function in (28) $\varphi=\left[\left(u_{\omega}\right)_{h}-k_{n}\right]_{+} \xi_{n}^{2}$ and integrate in time over $\left(t^{*}-\frac{R_{n}^{2}}{\phi_{2}\left(\frac{\omega}{4}\right)}, t\right)$ for $t \in\left(t^{*}-\frac{R_{n}^{2}}{\phi_{2}\left(\frac{\omega}{4}\right)}, t^{*}\right)$ with $K=B_{R_{n}}$. To simplify the notation, we put

$$
\tau_{n} \equiv t^{*}-\frac{R_{n}^{2}}{\phi_{2}\left(\frac{\omega}{4}\right)},
$$

and omit, from here on, $d x$ and $d t$ in all integrals.

We start with the lower order term since it encompasses the main novelty with respect to [26]. After passing to the limit in $h$, using the convergence properties of the Steklov average and Young's inequality, we obtain

$$
\begin{gathered}
\chi \int_{\tau_{n}}^{t} \int_{B_{R_{n}}} u f(u) \nabla v \cdot\left\{\xi_{n}^{2} \nabla\left(u_{\omega}-k_{n}\right)_{+}+2\left(u_{\omega}-k_{n}\right)_{+} \xi_{n} \nabla \xi_{n}\right\} \\
\leq \frac{1}{2} \phi_{2}\left(\frac{\omega}{4}\right) \int_{\tau_{n}}^{t} \int_{B_{R_{n}}}\left|\xi_{n} \nabla\left(u_{\omega}-k_{n}\right)_{+}\right|^{2}+\frac{1}{2 \phi_{2}\left(\frac{\omega}{4}\right)} M^{2} \int_{\tau_{n}}^{t} \int_{B_{R_{n}}}|\nabla v|^{2} \chi_{\left\{u_{\omega} \geq k_{n}\right\}} \\
+2 M \int_{\tau_{n}}^{t} \int_{B_{R_{n}}}|\nabla v|\left|\nabla \xi_{n}\right|\left(\frac{\omega}{4}\right) \chi_{\left\{u_{\omega} \geq k_{n}\right\}}
\end{gathered}
$$

since $\left(u_{\omega}-k_{n}\right)_{+} \leq \frac{\omega}{4}$, and defining $M:=\|\chi u f(u)\|_{L^{\infty}\left(Q_{T}\right)}$. Using again Young's inequality, we arrive at

$$
\begin{gathered}
\leq \frac{1}{2} \phi_{2}\left(\frac{\omega}{4}\right) \int_{\tau_{n}}^{t} \int_{B_{R_{n}}}\left|\xi_{n} \nabla\left(u_{\omega}-k_{n}\right)_{+}\right|^{2}+\frac{M^{2}+2 M}{2 \phi_{2}\left(\frac{\omega}{4}\right)} \int_{\tau_{n}}^{t} \int_{B_{R_{n}}}|\nabla v|^{2} \chi_{\left\{u_{\omega} \geq k_{n}\right\}} \\
+M \frac{2^{2(n+1)}}{R^{2}}\left(\frac{\omega}{4}\right)^{2} \phi_{2}\left(\frac{\omega}{4}\right) \int_{\tau_{n}}^{t} \int_{B_{R_{n}}} \chi_{\left\{u_{\omega} \geq k_{n}\right\}} .
\end{gathered}
$$


We conclude with the bound

$$
\int_{\tau_{n}}^{t} \int_{B_{R_{n}}}|\nabla v|^{2} \chi_{\left\{u_{\omega} \geq k_{n}\right\}} \leq\|\nabla v\|_{L^{p}\left(Q_{T}\right)}^{2}\left\{\int_{\tau_{n}}^{t}\left|A_{k_{n}, R_{n}}^{+}(\sigma)\right| d \sigma\right\}^{1-\frac{2}{p}}
$$

where $A_{k_{n}, R_{n}}^{+}(\sigma):=\left\{x \in B_{R_{n}}: u(x, \sigma)>k_{n}\right\}$ and $p$ is chosen sufficiently large. This is possible since $v \in L^{q}\left(0, T ; W^{2, q}(\Omega)\right)$, for all $q>1$.

The other terms are treated as in [26]; we repeat the main calculations here for the sake of completeness. The first term gives

$$
\begin{gathered}
\int_{\tau_{n}}^{t} \int_{B_{R_{n}}} \partial_{t}\left\{u_{h}\right\}\left[\left(u_{\omega}\right)_{h}-k_{n}\right]_{+} \xi_{n}^{2}=\frac{1}{2} \int_{\tau_{n}}^{t} \int_{B_{R_{n}}} \partial_{t}\left\{\left[\left(u_{\omega}\right)_{h}-k_{n}\right]_{+}^{2}\right\} \xi_{n}^{2} \\
+\left(1-\frac{\omega}{4}-k_{n}\right) \int_{\tau_{n}}^{t} \int_{B_{R_{n}}} \partial_{t}\left\{\left(\left[u-\left(1-\frac{\omega}{4}\right)\right]_{+}\right)_{h}\right\} \xi_{n}^{2} .
\end{gathered}
$$

Next, integrate by parts and let $h \rightarrow 0$. We get

$$
\begin{gathered}
\frac{1}{2} \int_{B_{R_{n}} \times\{t\}}\left(u_{\omega}-k_{n}\right)_{+}^{2} \xi_{n}^{2}-\frac{1}{2} \int_{B_{R_{n}} \times\left\{\tau_{n}\right\}}\left(u_{\omega}-k_{n}\right)_{+}^{2} \xi_{n}^{2} \\
-\int_{\tau_{n}}^{t} \int_{B_{R_{n}}}\left(u_{\omega}-k_{n}\right)_{+}^{2} \xi_{n} \partial_{t} \xi_{n}+\left(1-\frac{\omega}{4}-k_{n}\right)\left\{\int_{B_{R_{n}} \times\{t\}}\left[u-\left(1-\frac{\omega}{4}\right)\right]_{+} \xi_{n}^{2}\right. \\
\left.-\int_{B_{R_{n}} \times\left\{\tau_{n}\right\}}\left[u-\left(1-\frac{\omega}{4}\right)\right]_{+} \xi_{n}^{2}-2 \int_{\tau_{n}}^{t} \int_{B_{R_{n}}}\left[u-\left(1-\frac{\omega}{4}\right)\right]_{+} \xi_{n} \partial_{t} \xi_{n}\right\}=(*) .
\end{gathered}
$$

Since the second and the fifth terms vanish, due to the fact that $\xi_{n}$ was chosen such that it vanishes on the parabolic boundary of $Q_{R_{n}}^{t^{*}}$, and the fourth term is positive, we get, using the other assumptions on $\xi_{n}$,

$$
\begin{gathered}
(*) \geq \frac{1}{2} \int_{B_{R_{n}} \times\{t\}}\left(u_{\omega}-k_{n}\right)_{+}^{2} \xi_{n}^{2}-\phi_{2}\left(\frac{\omega}{4}\right) \frac{2^{2(n+1)}}{R^{2}}\left(\frac{\omega}{4}\right)^{2} \int_{\tau_{n}}^{t} \int_{B_{R_{n}}} \chi_{\left\{u_{\omega} \geq k_{n}\right\}} \\
-2 \phi_{2}\left(\frac{\omega}{4}\right) \frac{2^{2(n+1)}}{R^{2}}\left(\frac{\omega}{4}\right)^{2} \int_{\tau_{n}}^{t} \int_{B_{R_{n}}} \chi_{\left\{u \geq 1-\frac{\omega}{4}\right\}}=(* *) .
\end{gathered}
$$

Observe that $1-\frac{\omega}{4}-k_{n} \leq \frac{\omega}{4}$ and $\left[u-\left(1-\frac{\omega}{4}\right)\right]_{+} \leq \frac{\omega}{4}$. Finally, remarking that $u \geq 1-\frac{\omega}{4} \Rightarrow u_{\omega} \geq k_{n}$, we obtain

$$
(* *) \geq \frac{1}{2} \int_{B_{R_{n}} \times\{t\}}\left(u_{\omega}-k_{n}\right)_{+}^{2} \xi_{n}^{2}-3 \phi_{2}\left(\frac{\omega}{4}\right) \frac{2^{2(n+1)}}{R^{2}}\left(\frac{\omega}{4}\right)^{2} \int_{\tau_{n}}^{t} \int_{B_{R_{n}}} \chi_{\left\{u_{\omega} \geq k_{n}\right\}} .
$$


Concerning the diffusion term, we first pass to the limit in $h$, obtaining

$$
\begin{gathered}
\int_{\tau_{n}}^{t} \int_{B_{R_{n}}}(a(u) \nabla u)_{h} \cdot \nabla\left\{\left[\left(u_{\omega}\right)_{h}-k_{n}\right]_{+} \xi_{n}^{2}\right\} \\
\longrightarrow \int_{\tau_{n}}^{t} \int_{B_{R_{n}}} a(u) \nabla u \cdot\left\{\xi_{n}^{2} \nabla\left(u_{\omega}-k_{n}\right)_{+}+2\left(u_{\omega}-k_{n}\right)_{+} \xi_{n} \nabla \xi_{n}\right\} \\
=\int_{\tau_{n}}^{t} \int_{B_{R_{n}}} a(u)\left|\xi_{n} \nabla\left(u_{\omega}-k_{n}\right)_{+}\right|^{2}+2 \int_{\tau_{n}}^{t} \int_{B_{R_{n}}} a(u)\left(u_{\omega}-k_{n}\right)_{+} \xi_{n} \nabla u \cdot \nabla \xi_{n}=(*) .
\end{gathered}
$$

Next, we estimate the second term:

$$
\begin{gathered}
\left|2 \int_{\tau_{n}}^{t} \int_{B_{R_{n}}} a(u)\left(u_{\omega}-k_{n}\right)_{+} \xi_{n} \nabla u \cdot \nabla \xi_{n}\right| \\
\leq 2 \int_{\tau_{n}}^{t} \int_{B_{R_{n}}}|a(u)|\left(u_{\omega}-k_{n}\right)_{+} \xi_{n}\left|\nabla \xi_{n}\right|\left|\nabla\left(u_{\omega}-k_{n}\right)_{+}\right| \\
+\left|2\left(1-\frac{\omega}{4}-k_{n}\right) \int_{\tau_{n}}^{t} \int_{B_{R_{n}}} \xi_{n} \nabla\left\{\left(\int_{1-\frac{\omega}{4}}^{u} a(s) \mathrm{d} s\right)\right\} \cdot \nabla \xi_{n}\right| \\
\leq C_{1} \phi_{2}\left(\frac{\omega}{2}\right)\left\{\epsilon \int_{\tau_{n}}^{t} \int_{B_{R_{n}}}\left|\xi_{n} \nabla\left(u_{\omega}-k_{n}\right)_{+}\right|^{2}+\frac{1}{\epsilon} \int_{\tau_{n}}^{t} \int_{B_{R_{n}}}\left(u_{\omega}-k_{n}\right)_{+}^{2}\left|\nabla \xi_{n}\right|^{2}\right\} \\
+2\left(\frac{\omega}{4}\right)\left|-\int_{\tau_{n}}^{t} \int_{B_{R_{n}}}\left(\int_{1-\frac{\omega}{4}}^{u} a(s) \mathrm{d} s\right)_{+}\left(\left|\nabla \xi_{n}\right|^{2}+\xi_{n} \Delta \xi_{n}\right)\right| \\
\leq C_{1} \epsilon \phi_{2}\left(\frac{\omega}{2}\right) \int_{\tau_{n}}^{t} \int_{B_{R_{n}}}\left|\xi_{n} \nabla\left(u_{\omega}-k_{n}\right)_{+}\right|^{2}+\frac{C_{1} 2^{2(n+1)} \phi_{2}\left(\frac{\omega}{2}\right)}{\epsilon R^{2}}\left(\frac{\omega}{4}\right)^{2} \int_{\tau_{n}}^{t} \int_{B_{R_{n}}} \chi_{\left\{u_{\omega} \geq k_{n}\right\}} \\
+2\left(\frac{\omega}{4}\right) 2 \frac{2^{2(n+1)}}{R^{2}} \phi_{2}\left(\frac{\omega}{4}\right)\left(\frac{\omega}{4}\right) \int_{\tau_{n}}^{t} \int_{B_{R_{n}}} \chi_{\left\{u_{\omega} \geq k_{n}\right\}},
\end{gathered}
$$

since

$$
\left(\int_{1-\frac{\omega}{4}}^{u} a(s) \mathrm{d} s\right)_{+} \leq \phi_{2}\left(1-\left(1-\frac{\omega}{4}\right)\right)\left(1-\left(1-\frac{\omega}{4}\right)\right) .
$$

Observing that $\nabla\left(u_{\omega}-k_{n}\right)_{+}$is only nonzero in the set $\left\{k_{n}<u<1-\frac{\omega}{4}\right\}$, and that in this set

$$
a(u) \geq C_{0} \phi_{2}(1-u) \geq C_{0} \phi_{2}\left(1-\left(1-\frac{\omega}{4}\right)\right)=C_{0} \phi_{2}\left(\frac{\omega}{4}\right),
$$


we conclude, choosing $\epsilon=\frac{\left(C_{0}-1\right) \phi_{2}\left(\frac{\omega}{4}\right)}{C_{1} \phi_{2}\left(\frac{\omega}{2}\right)}$, that

$$
\begin{gathered}
\int_{\tau_{n}}^{t} \int_{B_{R_{n}}} a(u) \nabla u \cdot \nabla\left[\left(u_{\omega}-k_{n}\right)_{+} \xi_{n}^{2}\right] \geq \phi_{2}\left(\frac{\omega}{4}\right) \int_{\tau_{n}}^{t} \int_{B_{R_{n}}}\left|\xi_{n} \nabla\left(u_{\omega}-k_{n}\right)_{+}\right|^{2} \\
-\left\{\frac{\left[C_{1} \phi_{2}\left(\frac{\omega}{2}\right)\right]^{2}}{\left(C_{0}-1\right) \phi_{2}\left(\frac{\omega}{4}\right)}+4 \phi_{2}\left(\frac{\omega}{4}\right)\right\} \frac{2^{2(n+1)}}{R^{2}}\left(\frac{\omega}{4}\right)^{2} \int_{\tau_{n}}^{t} \int_{B_{R_{n}}} \chi_{\left\{u_{\omega} \geq k_{n}\right\}} .
\end{gathered}
$$

Now, putting the three estimates together, we arrive at

$$
\begin{gathered}
\operatorname{ess}_{\tau_{n} \leq t \leq t^{*}} \int_{B_{R_{n}} \times\{t\}}\left(u_{\omega}-k_{n}\right)_{+}^{2} \xi_{n}^{2}+\phi_{2}\left(\frac{\omega}{4}\right) \int_{\tau_{n}}^{t^{*}} \int_{B_{R_{n}}}\left|\xi_{n} \nabla\left(u_{\omega}-k_{n}\right)_{+}\right|^{2} \\
\leq 2\left\{\frac{\left[C_{1} \phi_{2}\left(\frac{\omega}{2}\right)\right]^{2}}{\left(C_{0}-1\right) \phi_{2}\left(\frac{\omega}{4}\right)}+(7+M) \phi_{2}\left(\frac{\omega}{4}\right)\right\} \frac{2^{2(n+1)}}{R^{2}}\left(\frac{\omega}{4}\right)^{2} \int_{\tau_{n}}^{t^{*}} \int_{B_{R_{n}}} \chi_{\left\{u_{\omega} \geq k_{n}\right\}} \\
+\frac{M^{2}+2 M}{\phi_{2}\left(\frac{\omega}{4}\right)}\|\nabla v\|_{L^{p}\left(Q_{T}\right)}^{2}\left\{\int_{\tau_{n}}^{t^{*}}\left|A_{k_{n}, R_{n}}^{+}(\sigma)\right| d \sigma\right\}^{1-\frac{2}{p}} .
\end{gathered}
$$

Next we perform a change in the time variable, putting $\bar{t}=\left(t-t^{*}\right) \phi_{2}\left(\frac{\omega}{4}\right)$, and define

$$
\overline{u_{\omega}}(\cdot, \bar{t})=u_{\omega}(\cdot, t) \quad \text { and } \quad \overline{\xi_{n}}(\cdot, \bar{t})=\xi_{n}(\cdot, t),
$$

to obtain the simplified inequality

$$
\begin{aligned}
& \left\|\left(\overline{u_{\omega}}-k_{n}\right)_{+} \overline{\xi_{n}}\right\|_{V_{0}^{2}\left(Q\left(R_{n}^{2}, R_{n}\right)\right)}^{2} \\
& \leq 2\left\{\frac{C_{1}^{2}}{\left(C_{0}-1\right)}\left[\frac{\phi_{2}\left(\frac{\omega}{2}\right)}{\phi_{2}\left(\frac{\omega}{4}\right)}\right]^{2}+7+M\right\} \frac{2^{2(n+1)}}{R^{2}}\left(\frac{\omega}{4}\right)^{2} A_{n} \\
& \quad+\left(M^{2}+2 M\right)\|\nabla v\|_{L^{p}\left(Q_{T}\right)}^{2}\left[\phi_{2}\left(\frac{\omega}{4}\right)\right]^{\frac{2}{p}-2} A_{n}^{1-\frac{2}{p}},
\end{aligned}
$$

defining, for each $n$,

$$
A_{n}=\int_{-R_{n}^{2}}^{0} \int_{B_{R_{n}}} \chi_{\left\{\overline{u_{\omega}} \geq k_{n}\right\}} \mathrm{d} x \mathrm{~d} \bar{t} .
$$

Next, observe that the following estimates hold

$$
\frac{1}{2^{2(n+2)}}\left(\frac{\omega}{4}\right)^{2} \quad A_{n+1} \leq\left|k_{n+1}-k_{n}\right|^{2} A_{n+1}
$$




$$
\begin{gathered}
\leq\left\|\left(\overline{u_{\omega}}-k_{n}\right)_{+}\right\|_{2, Q\left(R_{n+1}^{2}, R_{n+1}\right)}^{2} \\
\leq\left\|\left(\overline{u_{\omega}}-k_{n}\right)_{+} \overline{\xi_{n}}\right\|_{2, Q\left(R_{n}^{2}, R_{n}\right)}^{2} \\
\leq C\left\|\left(\overline{u_{\omega}}-k_{n}\right)_{+} \overline{\xi_{n}}\right\|_{V_{0}^{2}\left(Q\left(R_{n}^{2}, R_{n}\right)\right)}^{2} A_{n}^{\frac{2}{N+2}} \\
\leq 2 C\left\{\frac{C_{1}^{2}}{\left(C_{0}-1\right)}\left[\frac{\phi_{2}\left(\frac{\omega}{2}\right)}{\phi_{2}\left(\frac{\omega}{4}\right)}\right]^{2}+7+M\right\} \frac{2^{2(n+1)}}{R^{2}}\left(\frac{\omega}{4}\right)^{2} A_{n}^{1+\frac{2}{N+2}} \\
+C\left(M^{2}+2 M\right)\|\nabla v\|_{L^{p}\left(Q_{T}\right)}^{2}\left[\phi_{2}\left(\frac{\omega}{4}\right)\right]^{\frac{2}{p}-2} A_{n}^{1-\frac{2}{p}+\frac{2}{N+2}} .
\end{gathered}
$$

In fact, the first and the third inequalities are obvious; the second one holds due to the fact that $k_{n}<k_{n+1}$; the fourth inequality is a consequence of a well known imbedding theorem (see, e.g., corollary 3.1 on chapter I of [6]) and the last one follows from (33). Next, define the numbers

$$
X_{n}=\frac{A_{n}}{\left|Q\left(R_{n}^{2}, R_{n}\right)\right|} \quad ; \quad Z_{n}=\frac{A_{n}^{1 / p}}{\left|B_{R_{n}}\right|},
$$

divide (34) by $\left|Q\left(R_{n+1}^{2}, R_{n+1}\right)\right|$ and obtain the recursive relation

$$
X_{n+1} \leq \gamma 4^{2 n}\left\{X_{n}^{1+\frac{2}{N+2}}+X_{n}^{\frac{2}{N+2}} Z_{n}^{1+\kappa}\right\}, \quad n=0,1,2, \ldots
$$

where $\kappa=p-3>0$ and

$$
\gamma=C \max \left\{\frac{C_{1}^{2}}{\left(C_{0}-1\right)}\left[\frac{\phi_{2}\left(\frac{\omega}{2}\right)}{\phi_{2}\left(\frac{\omega}{4}\right)}\right]^{2}+7+M ; \quad\left(\frac{\omega}{4}\right)^{-2}\left[\phi_{2}\left(\frac{\omega}{4}\right)\right]^{\frac{2}{p}-2} R^{N \kappa}\right\} .
$$

A similar reasoning leads to

$$
Z_{n+1} \leq \gamma 4^{2 n}\left\{X_{n}+Z_{n}^{1+\kappa}\right\}, \quad n=0,1,2, \ldots
$$

We can now use a lemma on the fast geometric convergence of sequences (see lemma 4.2 on chapter I of [6]) to conclude that if

$$
X_{0}+Z_{0}^{1+\kappa} \leq(4 \gamma)^{-\frac{1+\kappa}{\theta}} 4^{\frac{-2(1+\kappa)}{\theta^{2}}} \equiv \nu_{0}, \quad \theta=\min \left\{\frac{2}{N+2} ; \kappa\right\}
$$

then

$$
X_{n}, Z_{n} \longrightarrow 0
$$


But (35) follows from the assumption (32) of the lemma and the conclusion is a consequence of (36). In fact, observe that

$$
R_{n} \searrow \frac{R}{2} \quad \text { and } \quad k_{n} \nearrow 1-\frac{\omega}{4},
$$

and since (36) implies that $A_{n} \rightarrow 0$, we conclude that

$$
\begin{gathered}
\left|\left\{(x, \bar{t}) \in Q\left(\left(\frac{R}{2}\right)^{2}, \frac{R}{2}\right): \overline{u_{\omega}}(x, \bar{t}) \geq 1-\frac{\omega}{4}\right\}\right| \\
=\left|\left\{(x, t) \in Q_{\frac{R}{2}}^{t^{*}}: u(x, t) \geq 1-\frac{\omega}{4}\right\}\right|=0
\end{gathered}
$$

and the lemma is proved.

It remains to show that $\nu_{0}$, i.e., $\gamma$, is independent of $\omega$, which is crucially related to the fact that $\phi_{2}$ is a power. In fact, we have

$$
\frac{\phi_{2}\left(\frac{\omega}{2}\right)}{\phi_{2}\left(\frac{\omega}{4}\right)}=\left(\frac{\omega}{2}\right)^{p_{2}}\left(\frac{4}{\omega}\right)^{p_{2}}=2^{p_{2}} .
$$

On the other hand, we can assume, without loss of generality, that

$$
\left(\frac{\omega}{4}\right)^{-2}\left[\phi_{2}\left(\frac{\omega}{4}\right)\right]^{\frac{2}{p}-2} R^{N \kappa} \leq 1 .
$$

Otherwise, we would have $\omega<C R^{\alpha}$, with $\alpha=\frac{N \kappa p}{2 p+2 p p_{2}-2 p_{2}}>0$, and the result would be trivial.

Our next aim is to show that the conclusion of Lemma 6 holds in a full cylinder of the type $Q(\tau, \rho)$. The idea is to use the fact that at the time level

$$
-\widehat{t}:=t^{*}-\frac{\left(\frac{R}{2}\right)^{2}}{\phi_{2}\left(\frac{\omega}{4}\right)}
$$

the function $u(x)$ is strictly below the level $1-\frac{\omega}{4}$ in the ball $B_{\frac{R}{2}}$ and look at this time level as an initial condition to make the conclusion hold up to $t=0$, eventually shrinking the ball. Again this is a sophisticated way of showing that somehow the equation behaves like the heat equation. As an intermediate step we need the following lemma.

Lemma 7. Given $\nu_{1} \in(0,1)$, there exists $s_{1} \in \mathbb{N}$, depending only on the data, such that

$$
\left|\left\{x \in B_{\frac{R}{4}}: u(x, t) \geq 1-\frac{\omega}{2^{s_{1}}}\right\}\right| \leq \nu_{1}\left|B_{\frac{R}{4}}\right| \quad, \quad \forall t \in(-\widehat{t}, 0) .
$$


The proof uses logarithmic estimates and the differences with respect to what is done in [26] are of the same nature as the ones described in the previous lemma. For that reason it will be omitted as well as the proof of the next result, that establishes the conclusion of the first alternative.

Proposition 1. There exist constants $\nu_{0} \in(0,1)$ and $1<s_{1} \in \mathbb{N}$, depending only on the data, such that if (32) holds then

$$
u(x, t)<1-\frac{\omega}{2^{s_{1}+1}}, \quad \text { a.e. } \quad(x, t) \in Q\left(\widehat{t}, \frac{R}{8}\right) .
$$

Corollary 1. There exist constants $\nu_{0}, \sigma_{0} \in(0,1)$, depending only on the data, such that if (32) holds then

$$
\underset{Q\left(\widehat{t}, \frac{R}{8}\right)}{\operatorname{ess}} \operatorname{osc} u \sigma_{0} \omega
$$

Proof: We can use Proposition 1 to obtain $s_{1} \in \mathbb{N}$ such that

$$
\underset{Q\left(\widehat{t}, \frac{R}{8}\right)}{\operatorname{ess} \sup } u \leq 1-\frac{\omega}{2^{s_{1}+1}}
$$

and from this we get

$$
\underset{Q\left(\widehat{t}, \frac{R}{8}\right)}{\operatorname{ess} \operatorname{OSc}} u=\underset{Q\left(\widehat{t}, \frac{R}{8}\right)}{\operatorname{ess} \sup } u-\underset{Q\left(\widehat{t}, \frac{R}{8}\right)}{\operatorname{ess} \inf } u \leq 1-\frac{\omega}{2^{s_{1}+1}}-0=\left(1-\frac{1}{2^{s_{1}+1}}\right) \omega
$$

and the corollary follows with $\sigma_{0}=\left(1-\frac{1}{2^{s_{1}+1}}\right)$.

The conclusion of the second alternative is entirely similar, the analysis being performed near the degeneracy at 0 , and we refer the reader to [26] for the details. The proof of Theorem 2 now follows from Corollary 1, and its alternative counterpart, through the usual method (cf. [7]) of defining recursively a sequence of nested and shrinking cylinders $Q_{n}$ and a sequence $\omega_{n}$ converging to zero such that

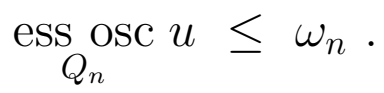

We stress that the Hölder continuity is obtained since $\sigma_{0}$ in Corollary 1 is independent of the oscillation $\omega$. 


\section{References}

[1] H.W. Alt and E. DiBenedetto, Nonsteady flow of water and oil through inhomogeneous porous media, Ann. Sc. Norm. Sup. Pisa (IV), 12 (1985), 335-392.

[2] H. Amann, Nonhomogeneous linear and quasilinear elliptic and parabolic boundary value problems, Function spaces, differential operators and nonlinear analysis, pp. 9-126, Teubner-Texte Math. 133, Teubner, Stuttgart, 1993.

[3] M. Bendahmane and K. H. Karlsen, Renormalized entropy solutions for quasilinear anisotropic degenerate parabolic equations, SIAM J. Math. Anal., 2 (2004), 405-422.

[4] M. Burger, M. Di Francesco, Y. Dolak-Struss, The Keller-Segel model for chemotaxis with prevention of overcrowding: linear vs. nonlinear diffusion, preprint.

[5] J. Carrillo, Entropy solutions for nonlinear degenerate problems, Arch. Rational Mech. Anal., 147(4), (1999):269-361.

[6] E. DiBenedetto, Degenerate Parabolic Equations, Springer-Verlag, New York, 1993.

[7] E. DiBenedetto, J.M. Urbano and V. Vespri, Current issues on singular and degenerate evolution equations, Handbook of Differential Equations, Evolutionary Equations, vol.1, pp. 169286, Elsevier/North-Holland, Amsterdam, 2004.

[8] E. Henriques and J.M. Urbano, On the doubly singular equation $\gamma(u)_{t}=\Delta_{p} u$, Comm. Partial Differential Equations, 30 (2005), 919-955.

[9] E. Henriques and J.M. Urbano, Intrinsic scaling for PDEs with an exponential nonlinearity, Indiana Univ. Math. J., to appear.

[10] M.A. Herrero and J.J.L. Velàzquez, Chemotactic collapse for the Keller-Segel model, J. Math. Biol., 35 (1996), 177-194.

[11] T. Hillen and K. Painter, Global existence for a parabolic chemotaxis model with prevention of overcrowding, Adv. in Appl. Math., 26 (2001), 280-301.

[12] H.J. Hwang, K. Kang, A. Stevens, Drift-diffusion limits of kinetic models for chemotaxis: a generalization. Discrete Contin. Dyn. Syst. Ser. B 5(2) (2005), 319-334.

[13] W. Jäger and S. Luckhaus, On explosion of solutions to a system of partial differential equations modelling chemotaxis, Trans. Amer. Math. Soc., 329 (1992), 819-824.

[14] K. H. Karlsen and N. H. Risebro, On the uniqueness and stability of entropy solutions of nonlinear degenerate parabolic equations with rough coefficients, Discrete Contin. Dyn. Syst., 9 (2003), 1081-1104.

[15] J. Keener and J. Sneyd, Mathematical Physiology, Springer-Verlag, New York, 1998.

[16] E.F. Keller and L.A. Segel, Initiation of slime mold aggregation viewed as an instability, J. Theor. Biol., 26 (1970), 399-415.

[17] E.F. Keller and L.A. Segel, Model for chemotaxis, J. Theor. Biol., 30 (1971), 225-234.

[18] O.A. Ladyzhenskaya, V. Solonnikov and N. Ural'ceva, Linear and quasi-linear equations of parabolic type, Transl. AMS 23, Providence, 1968.

[19] R. Landes, On the existence of weak solutions for quasilinear parabolic initial-boundary value problems, Proc. Roy. Soc. Edinburgh Sect. A, 89 (1981), 217-237.

[20] P. Laurençot and D. Wrzosek, A chemotaxis model with threshold density and degenerate diffusion, HYKE preprint no. 2004-167.

[21] J.-L. Lions, Quelques méthodes de résolution des problèmes aux limites non linèaires, Dunod, Paris, 1969.

[22] K. Osaki, T. Tsujikawa, A. Yagi and M. Mimura, Exponential attractor for a chemotaxisgrowth system of equations, Nonlinear Anal., 51 (2002), 119-144.

[23] S. N. Kružkov, First order quasi-linear equations in several independent variables, Math. USSR Sbornik, 10(2), (1970), 217-243. 
[24] S. N. Kružkov, Results concerning the nature of the continuity of solutions of parabolic equations, and some of their applications, Mat. Notes, 6 (2002), 517-523.

[25] J. Simon, Compact sets in the space $L^{p}(0, T ; B)$, Ann. Mat. Pura Appl. (4) 146 (1987), 65-96.

[26] J.M. Urbano, Hölder continuity of local weak solutions for parabolic equations exhibiting two degeneracies, Adv. Differential Equations 6 (2001), 327-358.

[27] A. Yagi, Norm behavior of solutions to a parabolic system of chemotaxis, Math. Japon., 45 (1997), 241-265.

Mostafa Bendahmane

Centre of Mathematics for Applichtions, University of Oslo, P.O. Box 1053, Blindern, $\mathrm{N}-0316$ OsLo, Norway

E-mail address: mostafab@math.uio.no

KenNeth H. KARLSEN

Centre of Mathematics for Applichtions, University of Oslo, P.O. Box 1053, Blindern, $\mathrm{N}-0316$ OsLo, Norway

AND

Department of Scientific Computing, Simula Research Laboratory, P.O.Box 134, N-1325

LYSAKER, NORWAY

E-mail address: kennethk@math.uio.no

José Miguel Urbano

Departamento de Matemática, Universidade de Coimbra, 3001-454 Coimbra, Portugal

E-mail address: jmurb@mat.uc.pt 